\title{
Antimicrobial resistance profiles of Escherichia coli and prevalence of extended-spectrum beta-lactamase-producing Enterobacteriaceae in calves from organic and conventional dairy farms in Switzerland
}

\author{
Magdalena Nüesch-Inderbinen ${ }^{1}$, Claudia Hänni ${ }^{1}$, Sonja Hartnack ${ }^{1}$, Katrin Zurfluh ${ }^{1}$, and \\ Roger Stephan ${ }^{1}$ \\ ${ }^{1}$ University of Zurich
}

February 10, 2022

\begin{abstract}
This study compared the antimicrobial resistance (AMR) among commensal Escherichia coli in the fecal microbiota of young calves raised on organic and on conventional dairy farms in Switzerland. Further, fecal carriage of extended-spectrum betalactamase (ESBL) producing Enterobacteriaceae was assessed for calves from both farming systems. Where possible, data on antimicrobial usage (AMU) were obtained. Antimicrobial susceptibility testing was performed on a total of 71 isolates using the disk diffusion method. ESBL producers were characterized by PCR-based multilocus sequence typing and sequencing of the blaESBL genes. Organically raised calves were significantly more likely to harbor E. coli that showed antimicrobial resistance to ampicillin (OR 2.78, 95\% CI 1.02-7.61, p=0.046), streptomycin (OR 3.22, 95\%CI 1.17-8.92, p=0.046), kanamycin (OR 11.3, 95\% CI 2.94-43.50, $\mathrm{p}<0.001$ ), and tetracycline (OR 3.25, 95\%, 95\%CI 1.13-9.31, p=0.028). Calves with reported AMU were significantly more likely to harbor E. coli with resistance to ampicillin (OR 3.91, 95\%CI 1.03-14.85, p=0.045), streptomycin (OR 4.35, 95\% CI 1.13-16.7, p=0.045), and kanamycin (OR 8.69 95\%CI 2.01-37.7, p=0.004). ESBL-producing Enterobacteriaceae (18 E. coli and 3 Citrobacter braakii) were detected exclusively among samples from conventionally farmed calves (OR infinity ([?]), 95\%CI 2.3-[?], $\mathrm{p}<0.0013)$. The observations from this study suggest that AMR is highly prevalent among commensal E. coli in young dairy calves, irrespective of the farm management system, with proportions of certain resistance phenotypes higher among organic calves. By contrast, the occurrence of ESBL producers among young dairy calves may be linked to factors associated with conventional farming.
\end{abstract}

\section{Hosted file}

MB032021120545.pdf available at https://authorea.com/users/459918/articles/556023antimicrobial-resistance-profiles-of-escherichia-coli-and-prevalence-of-extendedspectrum-beta-lactamase-producing-enterobacteriaceae-in-calves-from-organic-andconventional-dairy-farms-in-switzerland 